\title{
Non-Renormalization Properties of the Chern-Simons Action Coupled to Matter
}

\author{
A. Blasin \\ Laboratoire d'Annecy-le-Vieux de Physique de Particules \\ Chemin de Bellevue BP 110, F - 74941 Annecy-le-Vieux Cedex, France \\ N. Maggiore \\ Dipartimento di Fisica - Università di Genova \\ Istituto Nazionale di Fisica Nucleare - sez. di Genova \\ Via Dodecaneso, 33 - 16146 Genova (Italy) \\ and \\ S. P. Sorellap \\ Département de Physique Théorique, Université de Genève \\ CH-1211 Genève 4, Switzerland
}

\begin{abstract}
We analyze an abelian gauge model in 3 dimensions which includes massless scalar matter fields. By controlling the trace anomalies with a local dilatation Ward identity, we show that, in perturbation theory and within the BPHZL scheme, the Chern-Simons term has no radiative corrections. This implies, in particular, the vanishing of the corresponding $\beta$ function in the renormalization group equation.
\end{abstract}

GEF-Th-7/1992

ENSLAPP-A-380-92

April 1992

\footnotetext{
${ }^{1}$ On leave of absence from Dipartimento di Fisica - Universitá di Trento, 38050 Povo (Trento) - (Italy)

${ }^{2}$ Supported in part by the Swiss National Science Foundation
} 


\section{Introduction}

The topological nature of the Chern-Simons action in three space-time dimensions leads naturally to the conjecture that it should be insensitive to local deformations such as the ones induced by the perturbative renormalization process of a local quantum field theory. That this is indeed so for a pure, non abelian gauge field theory, was first confirmed by explicit calculations [1, 2] and later proven to all perturbative orders [3], 田].

Although the techniques of the proofs adopted in this case are not directly extendible to a gauge field interacting with matter, in particular when scalar fields are present [], again the explicit low order calculations confirm that the Chern-Simons coupling constant has vanishing $\beta$-function [5, 6].

Here we propose a proof of this assertion, valid to all perturbative orders, which is based on local dilatation invariance ( general coordinate transformations in the Weyl's scheme ) as discussed in [0]. The idea of the proof is that the trace anomalies affecting the local dilatation Ward identity can be controlled, in the flat limit, by a single dimensionless scalar external field $\sigma(x)$, whose infinite couplings are all identified by those appearing in the classical $\sigma$-independent action.

Now the Chern-Simons term has the peculiarity of not being locally gauge invariant, its variation being a total divergence. Therefore, in this scheme where gauge and local dilatation invariance can be imposed independently, it follows that this term does not generate $\sigma$-field counterterms at any order and thus cannot contribute to the trace anomaly.

As expected this leads directly to the conclusion that the corresponding $\beta$-function vanishes. Moreover, in the course of the proof we shall also verify the stronger result that the Chern-Simons coupling does not renormalize at all.

This is also an expected consequence of the topological nature of the coupling which does not allow an identification in terms of a local nor-

\footnotetext{
${ }^{3}$ The case of only gauge and fermions fields can be treated with a slight extension of the method of ref [7], indeed it is well known that fermion fields without Yukawa couplings are cohomologically trivial [8].
} 
malization condition; hence the only way out is, in a local quantum field theory context, a complete insensitivity to radiative corrections.

For simplicity we shall treat the case of an abelian gauge field interacting with a massless scalar field $\phi$ with a $\left(\phi^{\dagger} \phi\right)^{3}$ self-interaction. The discussion proceeds within the BPHZL [9] regularization independent scheme; a more complete analysis including the Yang-Mills term as well as massive scalar and fermion fields is in preparation [10].

\section{The symmetries of the model}

The classical action in the Landau gauge is

$$
\begin{aligned}
\Sigma=\int d^{3} x( & \frac{k}{2} \varepsilon^{\mu \nu \rho} A_{\mu} \partial_{\nu} A_{\rho}-\left(D_{\mu} \phi\right)^{\dagger}\left(D^{\mu} \phi\right)-\frac{\lambda}{36}\left(\phi^{\dagger} \phi\right)^{3} \\
& \left.+\partial_{\mu} d A^{\mu}-\partial_{\mu} \bar{c} \partial^{\mu} c+i \gamma^{\dagger} \phi c-i \gamma \phi^{\dagger} c\right)
\end{aligned}
$$

where

$$
D_{\mu} \phi=\partial_{\mu} \phi-i A_{\mu} \phi
$$

The $B R S$ transformations are encoded in the nonlinear Slavnov identity

$$
\mathcal{S}(\Sigma)=\int d^{3} x\left(\partial_{\mu} c \frac{\delta \Sigma}{\delta A_{\mu}}+d \frac{\delta \Sigma}{\delta \bar{c}}+\frac{\delta \Sigma}{\delta \gamma^{\dagger}} \frac{\delta \Sigma}{\delta \phi}+\frac{\delta \Sigma}{\delta \gamma} \frac{\delta \Sigma}{\delta \phi^{\dagger}}\right)=0 .
$$

The action $\Sigma([2.1)$ satisfies the constraints:

$$
\begin{gathered}
\frac{\delta \Sigma}{\delta d}=-\partial A, \quad \frac{\delta \Sigma}{\delta \bar{c}}=\partial^{2} c \\
\frac{\delta \Sigma}{\delta c}=-\partial^{2} \bar{c}-i \gamma^{\dagger} \phi+i \gamma \phi^{\dagger} .
\end{gathered}
$$

The renormalizations compatible with (2.3), (2.4) and (2.5) are:

$$
\begin{aligned}
& k \rightarrow Z_{k} k \\
& \lambda \rightarrow Z_{\lambda} \lambda \\
& \phi \rightarrow Z \phi \\
& \gamma^{\dagger} \rightarrow Z^{-1} \gamma^{\dagger} .
\end{aligned}
$$


Notice that the ghost fields as well as the lagrangian multipliers do not renormalize due to the conditions $([2.4),(2.5)$ which identify uniquely the gauge-fixing part of the action.

To include the local dilatations [0] we have to introduce a metric $g^{\mu \nu}(x)=$ $\delta^{\mu \nu}+h^{\mu \nu}(x)$, a connection $\omega^{\mu}$ and the transformations laws with parameters $\lambda^{\rho}(x)$ :

$$
\begin{aligned}
& \delta g^{\mu \nu}=\lambda^{\rho} \partial_{\rho} g^{\mu \nu}-g^{\rho \nu} \partial^{\mu} \lambda_{\rho}-g^{\rho \mu} \partial^{\nu} \lambda_{\rho}+\frac{2}{3} g^{\mu \nu} \partial \lambda \\
& \delta \omega_{\mu}=\lambda^{\rho} \partial_{\rho} \omega_{\mu}+\omega_{\rho} \partial_{\mu} \lambda^{\rho}-\frac{1}{6} \partial_{\mu} \partial \lambda \\
& \delta A_{\mu}=\lambda^{\rho} \partial_{\rho} A_{\mu}+A_{\rho} \partial_{\mu} \lambda^{\rho} \\
& \delta \phi=\lambda^{\rho} \partial_{\rho} \phi+\frac{1}{6} \phi \partial \lambda \\
& \delta d=\lambda^{\rho} \partial_{\rho} d+\frac{1}{3} d \partial \lambda \\
& \delta \bar{c}=\lambda^{\rho} \partial_{\rho} \bar{c}+\frac{1}{3} \bar{c} \partial \lambda \\
& \delta c=\lambda^{\rho} \partial_{\rho} c \\
& \delta \gamma=\lambda^{\rho} \partial_{\rho} \gamma+\frac{5}{6} \gamma \partial \lambda .
\end{aligned}
$$

The action (2.1) becomes invariant under the above transformations with the replacements

$$
\begin{aligned}
& \delta^{\mu \nu} \rightarrow g^{\mu \nu} \\
& \partial_{\mu} \phi \rightarrow\left(\partial_{\mu}+\omega_{\mu}\right) \phi \\
& \partial_{\mu} d \rightarrow\left(\partial_{\mu}+2 \omega_{\mu}\right) d \\
& \partial_{\mu} \bar{c} \rightarrow\left(\partial_{\mu}+2 \omega_{\mu}\right) \bar{c} .
\end{aligned}
$$

We will only be interested in the flat limit $g^{\mu \nu} \rightarrow \delta^{\mu \nu}, \omega \rightarrow 0$ invariance which we write as

$$
\left(W_{\rho}(x) \Sigma(\omega, g)\right)_{F . L}=0 .
$$

Due to the choice of the Weyl weights in (2.7) the $B R S$ and $\delta$ symmetries commute so that they can be discussed independently.

As analyzed in detail in Ref. [0], the Weyl representation is unstable under radiative corrections and the ensuing trace anomalies, in the flat limit, can be reabsorbed by a single dimensionless external fields $\sigma(x)$ which is $B R S$ invariant and behaves as

$$
\delta \sigma(x)=\lambda^{\rho} \partial_{\rho} \sigma(x)+\frac{\hbar}{3} \partial \lambda \sigma(x) .
$$

The new Ward operator becomes

$$
\mathcal{W}_{\rho}(x)=W_{\rho}(x)+\partial_{\rho} \sigma(x) \frac{\delta}{\delta \sigma(x)}-\frac{\hbar}{3} \partial_{\rho} \frac{\delta}{\delta \sigma(x)},
$$


which still commutes with the $B R S$ transformations.

The introduction of the $\sigma$ field forces us to consider the vertex functional $\Gamma$ of the model as a double formal power series in $\sigma$ and $\hbar$ with coefficients $\Gamma^{(n, m)}$ at the order $\hbar^{n} \sigma^{m}$. The infinite number of $\sigma$-couplings do not correspond however to independent parameters; as shown in [7] they are recursively determined by the local dilatation Ward identity in the flat limit in terms of the $\sigma$ independent ones.

Two remark are now in order; first and foremost, the Chern-Simons term cannot couple to the $\sigma$ field since its $B R S$ variation is a divergence. Second, the presence of the $\sigma$ field does not spoil the supplementary conditions in (2.4), (2.5) which acquire $\sigma$ dependent terms on the right-hand side but which remain linear in the quantized fields. We can therefore summarize the results thus far obtained by saying that in the flat limit we can renormalize both the $B R S$ symmetry and the local dilation Ward identity by allowing a $\sigma$ dependence for the $Z, Z_{\lambda}$ renormalization constants, but not for $Z_{k}$. The effective action, in the sense of Zimmermann [9], is therefore

$$
\begin{aligned}
\Gamma^{e f f}=\int d^{3} x( & \frac{k}{2} Z_{k} \varepsilon^{\mu \nu \rho} A_{\mu} \partial_{\nu} A_{\rho}-\left(D_{\mu} Z(\sigma) \phi\right)^{\dagger}\left(D^{\mu} Z(\sigma) \phi\right) \\
& -\frac{\lambda}{36}\left(Z_{\lambda}(\sigma) Z_{\lambda}(\sigma)^{\dagger} \phi^{\dagger} \phi\right)^{3}+\partial_{\mu} d A^{\mu}-\partial_{\mu} \bar{c} \partial^{\mu} c \\
& \left.+i \gamma^{\dagger} \phi c-i \gamma \phi^{\dagger} c\right)
\end{aligned}
$$

where the free parameters are identified by the normalization conditions:

$$
\begin{gathered}
\left.\left.i \varepsilon_{\mu \nu \rho} \frac{p^{\rho}}{p^{2}} \frac{\delta^{2} \Gamma}{\delta \widehat{A}_{\mu}(p) \delta A_{\nu}(0)}\right|_{A=\phi=0, p^{2}=\mu^{2}} \equiv \Gamma_{A^{2}}\left(p^{2}\right)\right|_{p^{2}=\mu^{2}}=k \\
\left.\frac{\delta^{2} \Gamma}{\delta \hat{\phi}^{\dagger}(p) \delta \phi(0)}\right|_{A=\phi=0, p^{2}=\mu^{2}}=-\mu^{2} \\
\left.\frac{\delta^{6} \Gamma}{\delta \hat{\phi}^{\dagger}(p 1) \delta \hat{\phi}^{\dagger}(p 2) \delta \hat{\phi}^{\dagger}(p 3) \delta \hat{\phi}(p 4) \delta \hat{\phi}(p 5) \delta \phi(0)}\right|_{A=\phi=0, \text { symm. point } \mu^{2}}=-\lambda
\end{gathered}
$$

where the hat denotes the Fourier transform. 


\section{The renormalization group equation and the local dilatation invariance}

Having identified the parameters in (2.13) $-(2.15)$ we can write immediately a renormalization group equation for the vertex functional $\Gamma$ at $\sigma=0$; indeed a basis of Slavnov invariant differential vertex operator is provided by

$\left(\frac{\partial}{\partial k}, \quad \frac{\partial}{\partial \lambda}, \quad \mathcal{N}=\int d^{3} x\left(\phi \frac{\delta}{\delta \phi}-\gamma^{\dagger} \frac{\delta}{\delta \gamma^{\dagger}}\right), \quad \mathcal{N}^{\dagger}=\int d^{3} x\left(\phi^{\dagger} \frac{\delta}{\delta \phi^{\dagger}}-\gamma \frac{\delta}{\delta \gamma}\right)\right)$,

so that we have

$$
\left(\mu \frac{\partial}{\partial \mu}+\beta_{k} \frac{\partial}{\partial k}+\beta_{\lambda} \frac{\partial}{\partial \lambda}+\gamma_{\phi}\left(\mathcal{N}+\mathcal{N}^{\dagger}\right)\right) \Gamma=0
$$

where the coefficients $\beta_{k}, \beta_{\lambda}, \gamma_{\phi}$ can be computed with the help of the normalization conditions $(2.13)-(2.14)$, in particular we have

$$
\left.\left(\mu \frac{\partial \Gamma_{A^{2}}\left(p^{2}\right)}{\partial \mu}\right)\right|_{p^{2}=\mu^{2}}=-\beta_{k} .
$$

In order to establish a connection with the Ward identity

$$
\left(\mathcal{W}_{\rho}(x) \Gamma\right)_{\text {Flat-limit }}=0
$$

let us consider the insertionf

$$
\left.\left.\partial_{\sigma} \Gamma(\sigma)\right|_{\sigma=0} \equiv \int d^{3} x \frac{\delta \Gamma(\sigma)}{\delta \sigma(x)}\right|_{\sigma=0} .
$$

Moreover, by integrating the Ward operator in (2.11) with parameter $\lambda^{\rho}=$ $x^{\rho}$ we obtain

$$
\left(\mathcal{D}+\hbar \partial_{\sigma}\right) \Gamma(\sigma)=0
$$

where

$$
\mathcal{D}=\sum_{\psi} \int d^{3} x\left(x^{\rho} \partial_{\rho} \psi+d_{\psi}\right) \frac{\delta}{\delta \psi},
$$

\footnotetext{
${ }^{4}$ This insertion is well defined if the model has no infrared problems, which is also assumed in deriving the renormalization group equation; otherwise we remain with the local dilation Ward identity which controls the trace anomalies.
} 
and $\psi$ denotes collectively the $\left(A_{\mu}, \phi, \phi^{\dagger}\right)$ fields with canonical dimension $d_{\psi}=\left(1, \frac{1}{2}, \frac{1}{2}\right)$

By considering (3.6) at $\sigma=0$ we immediately find

$$
\mu \frac{\partial \Gamma}{\partial \mu}=\left.\hbar \partial_{\sigma} \Gamma(\sigma)\right|_{\sigma=0} .
$$

Let us now consider in detail the scaling behaviour imposed by (3.6) on the coupling $\Gamma_{A^{2}}\left(p^{2}\right)$ at the various orders in $\sigma$ and $\hbar$, with the constraint that the effective action in (2.12) does not contain $\sigma$ field couplings with the Chern-Simons term.

In Fourier transform we obtain for $\Gamma_{A^{2}}^{(n, m)}\left(p^{2}\right)$ the recursive relation

$$
p_{\rho} \frac{\partial \Gamma_{A^{2}}^{(n, m)}}{\partial p_{\rho}}+\Gamma_{A^{2}}^{(n-1, m+1)}=0 .
$$

Recalling that $\Gamma_{A^{2}}^{e f f(n, m)}=0$ for $m \geq 1$, and using the Ward identity (3.4), we shall be able to identify the general solution of $\Gamma_{A^{2}}^{(n, 0)}\left(p^{2}\right)$ as a function of $p^{2}$ parametrized with coefficients which are dependent on the dimensionless constant $\kappa$ and on $\ln \mu^{2}$; then we shall employ the normalization condition (2.13) and the power counting in order to find the $\mu^{2}$ dependence of $Z_{k}$.

For $n=1, m=0$ we get

$$
p_{\rho} \frac{\partial \Gamma_{A^{2}}^{(1,0)}\left(p^{2}\right)}{\partial p_{\rho}}=0
$$

hence

$$
\Gamma_{A^{2}}^{(1,0)}=\alpha_{0}^{1},
$$

where $\alpha_{0}^{1}$ is a $p^{2}$-independent constant.

At the next order $n=2, m=0$ we obtain

$$
p_{\rho} \frac{\partial \Gamma_{A^{2}}^{(2,0)}}{\partial p_{\rho}}+\Gamma_{A^{2}}^{(1,1)}=0,
$$


and also, for $n=1, m=1$

$$
p_{\rho} \frac{\partial \Gamma_{A^{2}}^{(1,1)}}{\partial p_{\rho}}=0
$$

hence

$$
\Gamma_{A^{2}}^{(1,1)}\left(p^{2}\right)=\alpha_{1}^{1}, \quad \Gamma_{A^{2}}^{(2,0)}\left(p^{2}\right)=-\frac{1}{2} \alpha_{1}^{1} \ln \left(p^{2}\right)+\alpha_{0}^{2} .
$$

Proceeding inductively we find

$$
\Gamma_{A^{2}}^{(n, 0)}\left(p^{2}\right)=\sum_{j=0}^{n-1}\left(-\frac{1}{2}\right)^{j} \alpha_{j}^{n-j}\left(\ln p^{2}\right)^{j}, \quad n \geq 1
$$

where the $\alpha_{j}^{n-j}$ are functions of $\ln \mu^{2}$ and $\kappa$. Now the power counting properties of $\Gamma_{A^{2}}^{(n, 0)}\left(p^{2}\right)$ imply that the above expression can be rewritten as

$$
\Gamma_{A^{2}}^{(n, 0)}\left(p^{2}\right)=\sum_{j=0}^{n-1} \gamma_{j}^{n}\left(\ln \frac{p^{2}}{\mu^{2}}\right)^{j}, \quad n \geq 1
$$

where the $\gamma_{j}^{n}$ are $\mu^{2}$ independent and uniquely determine the $\ln \mu^{2}$ dependence of the $\alpha_{j}^{n-j}$ by

$$
\left(-\frac{1}{2}\right)^{j} \alpha_{j}^{n-j}=\sum_{r=j}^{n-1} \gamma_{r}^{n}\left(-\ln \mu^{2}\right)^{r-j} \frac{j !}{(r-j) ! r !}, \quad n \geq 1 .
$$

The normalization condition (2.13) yields

$$
\gamma_{0}^{n}=0, \quad n \geq 1
$$

so that the perturbative power series for the $\beta_{k}$ and the $Z_{k}^{(n)}$ renormalization constant are given, in terms of (3.16) by

$$
\beta_{k}^{(1)}=0, \beta_{k}^{(n)}=\gamma_{1}^{n}, Z_{k}^{(1)}=0, Z_{k}^{(n)}=\sum_{j=1}^{n-1} \gamma_{j}^{n}\left(-\ln \mu^{2}\right)^{j}, \quad n \geq 2 .
$$

In particular we see that the one loop $\beta_{k}$ function is identically zero and that the vanishing of the higher orders is related to the vanishing of the $\gamma_{1}^{n}$ coefficients. 


\section{Non-renormalization of the Chern-Simons term}

In order to prove that the $Z_{k}^{(n)}$ 's vanish or equivalently that all $\gamma_{k}^{n}$ also do so, we employ relations (3.5), (3.19) and the Quantum Action Principle [1]].

Now all the Slavnov invariant, $\sigma$ dependent insertions are deducible from the effective action in (2.12); expanding the renormalization constants $Z_{\lambda}$, $Z$ to first order in $\sigma$

$$
\begin{aligned}
Z_{\lambda}(\sigma) & =Z_{\lambda}^{(0)}+\sigma Z_{\lambda}^{(1)}+O\left(\sigma^{2}\right) \\
Z(\sigma) & =Z^{(0)}+\sigma Z^{(1)}+O\left(\sigma^{2}\right),
\end{aligned}
$$

and considering the basis of insertions

$$
\begin{aligned}
\Delta_{C S} & =\frac{1}{2} \int d^{3} x \varepsilon^{\mu \nu \rho} A_{\mu} \partial_{\nu} A_{\rho}, \\
\Delta_{\lambda} & =-\frac{1}{36} \int d^{3} x\left(\phi^{\dagger} \phi\right)^{3} \\
\Delta & =-\int d^{3} x\left(D_{\mu} \phi\right)^{\dagger}\left(D^{\mu} \phi\right),
\end{aligned}
$$

we find

$$
\begin{aligned}
& \left.\left(\mu \frac{\partial \mathcal{Z}}{\partial \mu}\right)\right|_{\sigma=0}=\left.\frac{1}{\hbar}\left(\mu \frac{\partial \Gamma^{e f f}}{\partial \mu} \cdot \mathcal{Z}\right)\right|_{\sigma=0} \\
& =\left.\left(k \mu \frac{\partial Z_{k}}{\partial \mu} \Delta_{C S}+\mu \frac{\partial\left(Z^{(0)} Z^{(0) \dagger}\right)}{\partial \mu} \Delta+\mu \frac{\partial\left(Z_{\lambda}^{(0)}\left(Z^{(0)} Z^{(0) \dagger}\right)^{3}\right)}{\partial \mu} \Delta_{\lambda}\right) \cdot \mathcal{Z}\right|_{\sigma=0},
\end{aligned}
$$

and likewise

$$
\begin{aligned}
& \left.\left(\hbar \frac{\partial \mathcal{Z}}{\partial \sigma}\right)\right|_{\sigma=0}=\left.\left(\frac{\partial \Gamma^{e f f}}{\partial \sigma} \cdot \mathcal{Z}\right)\right|_{\sigma=0} \\
& =\left.\left(\left(Z^{(1)}+Z^{(1) \dagger}\right) \Delta+\left(3 Z_{\lambda}^{(0)}\left(Z^{(1)}+Z^{(1) \dagger}\right)+Z_{\lambda}^{(1)}\left(Z^{(0)} Z^{(0) \dagger}\right)^{3}\right) \Delta_{\lambda}\right) \cdot \mathcal{Z}\right|_{\sigma=0},
\end{aligned}
$$

where $\mathcal{Z}$ denotes the disconnected functional whose connected part $\mathcal{Z}_{c}$ is given by the Legendre transform of the the vertex functional $\Gamma$ :

$$
\mathcal{Z}_{c}(J, \sigma)=\Gamma(\sigma)+\sum_{\varphi=\text { all fields }} \int d^{3} x J \varphi .
$$

Since the left hand sides of (4.3), (4.4) coincide, and we have expandend on an independent basis, we obtain the relation

$$
\mu \frac{\partial Z_{k}}{\partial \mu}=0
$$


which, substituing (3.19), implies recursively

$$
\gamma_{j}^{n}=0, \quad n \geq 1 .
$$

The proof presented here proceeds straightforwardly once the main point, i.e. the lack of local gauge invariance of the Chern-Simons term is recognized as the property which accounts for the absence of the corresponding trace anomalies. Clearly a similar method can be applied to discuss generalizations of this model, for instance the inclusions of massive scalars, fermions with Yukawa coupling etc.

Acknowledgments: We would like to thank O. Piguet for discussions on the subject of this paper; one of the authors (A.B.) would like to thank the ENSLAPP of Annecy-le-Vieux for hospitality.

\section{References}

[1] E. Guadagnini, M. Martellini and M. Mintchev, Nucl. Phys. B336 (1990) 581;

L. Alvarez-Gaume, J. M. F. Labastida and A. V. Ramallo, Nucl. Phys. B334 (1990) 103;

[2] D. Birmingham, M. Rakowski and G. Thompson, Nucl. Phys. B329 (1990) 83;

[3] A. Blasi and R. Collina, Nucl. Phys. B345 (1990) 472;

[4] F. Delduc, O. Piguet, C. Lucchesi and S. P. Sorella, Nucl. Phys. B346 (1990) 313;

[5] S. Coleman and B. Hill, Phys. Lett. B159 (1985) 184;

G. Semenoff, P. Sodano and Yong-Shi Wu, Phys. Rev. Lett. 62 (1989) 715;

[6] L. V. Avdeev, G. V. Grigorev and D. I. Kazakov, " Renormalization in abelian Chern-Simons field theories with matter. ", CERNTH.6091/91 
[7] G. Bandelloni, C. Becchi, A. Blasi and R. Collina, Nucl. Phys. B197 (1982) 347;

C. Becchi, A. Blasi and R. Collina, Nucl. Phys. B274 (1986) 121;

[8] O. Piguet and A. Rouet, Phys. Reports 76 (1981) 1;

[9] J. H. Lowenstein and W. Zimmermann, Comm. Math. Phys. 44 (1975) 73;

J. H. Lowenstein , Comm. Math. Phys. 47 (1976) 53;

[10] A. Blasi, N. Maggiore and S. P. Sorella, in prep.

[11] Y.M.P Lam, Phys. Rev. D6 (1972) 2145, 2161;

T.E. Clark and J.H. Lowenstein, Nucl. Phys. B113 (1976) 109; 